Europhys. Lett., 57 (5), pp. 651-657 (2002)

\title{
Multiple-path interferometer with a single quantum obstacle
}

\author{
H. Schomerus ${ }^{1}$, Y. Noat ${ }^{2}$, J. Dalibard ${ }^{3}$ and C. W. J. Beenakker ${ }^{4}$ \\ 1 Max-Planck-Institut für Physik komplexer Systeme \\ Nöthnitzer Str. 38, $0118^{7}$ Dresden, Germany \\ 2 Kamerlingh Onnes Laboratory, Universiteit Leiden \\ P.O. Box 9504, 2300 RA Leiden, The Netherlands \\ 3 Laboratoire Kastler Brossel, Département de Physique de l'Ecole Normale Supérieure \\ 24 rue Lhomond, 75231 Paris, France \\ 4 Instituut-Lorentz, Universiteit Leiden, P.O. Box 9506 \\ 2300 RA Leiden, The Netherlands
}

(received 22 August 2001; accepted in final form 14 December 2001)

PACS. 05.60.Gg - Quantum transport.

PACS. 03.65. Nk - Scattering theory.

PACS. $42.25 . \mathrm{Hz}$ - Interference.

\begin{abstract}
We consider the scattering of particles (kinetic energy $\varepsilon$ ) by an obstacle which tunnels coherently between two positions (tunnel splitting $\Delta$ ), for arbitrary values of $\varepsilon / \Delta$ and scattering strength $U$. The obstacle mimics two classical scatterers at fixed positions when $\varepsilon \lesssim \Delta$. Interference disappears when $\varepsilon \gg \Delta$, but can be recovered if the elastic-scattering channel is detected only. At intermediate values of $\varepsilon$ there is a systematic interplay of the coherent tunnel motion and the ballistic particle motion. We show that the transmission probability can remain finite even in the limit $U \rightarrow \infty$ because the particle can evade the obstacle systematically. We discuss the realization of a quantum obstacle in mesoscopic systems.
\end{abstract}

In the familiar double-slit experiment a beam of particles is sent through two slits in a plate and the transmitted intensity is observed on a screen. One finds an interference pattern, thus demonstrating the coherent superposition of the two possible scattering paths. The same result is obtained for the reflection from obstacles, e.g., the bars of a reflection grating.

In these experiments the slits or bars only play a passive role. In this paper we consider scattering by a single obstacle, which, however, is by itself a quantum object with states that correspond to different locations (see fig. 1). Allowing for superpositions of these states, we have an obstacle that is delocalized in space. Which reflection or transmission pattern is then observed on the screen? It is straightforward to demonstrate that the quantum obstacle may act as a collection of classical scatterers, in that one observes the corresponding interference pattern on the screen. The condition for interference is that the tunnel splitting $\Delta$ is larger than the kinetic energy $\varepsilon$ of the incident particles (fast-tunneling regime). For $\Delta \ll \varepsilon$ (slowtunneling regime) the interference pattern from the obstacle disappears, but can be recovered if only the elastic-scattering channel is detected.

(c) EDP Sciences 


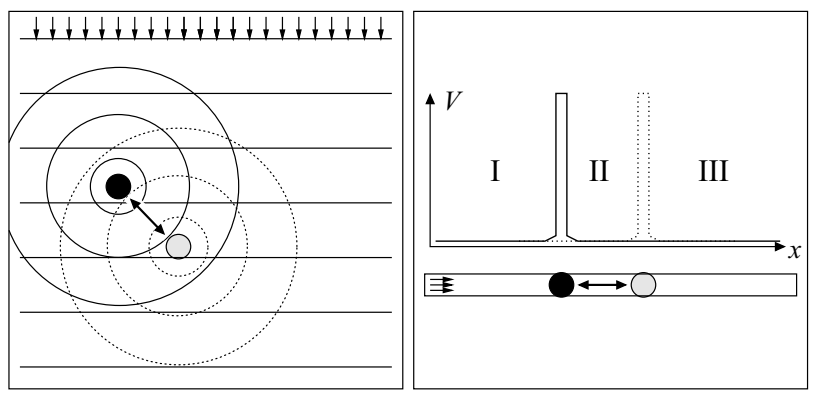

Fig. 1 - Scattering of a particle by a delocalized obstacle which tunnels between two positions (solid and shaded dot). Left panel: double slit, the obstacle provides two scattering paths in parallel. The lines indicate fronts of the wave function. Right panel: Fabry-Perot interferometer, the obstacle's positions are arranged in series. The solid and dashed curves indicate the scattering potentials. The problem is solved by matching the waves in the regions I, II, and III.

In this paper we present a one-dimensional model with repulsive contact interaction (deltafunction potential $U \delta(x)$ ) which is exactly solvable for arbitrary values of $\varepsilon / \Delta$ and $U$ and hence allows to study the crossover regime $\Delta \lesssim \varepsilon$ between the two regimes discussed above (we mostly concentrate on the case of large interaction strength $U$, in which the phenomena are most pronounced). The double slits or bars are two scattering elements put in parallel. Our model addresses the problem of two barriers arranged in series - a Fabry-Perot interferometer. The quantum analogue is an obstacle which tunnels between two locations along the propagation direction of the incident particles.

In the context of our model, interference results in transmission resonances. We find that resonances are absent in the slow-tunneling regime, while in the fast-tunneling regime the transmission amplitude becomes identical to that of the Fabry-Perot interferometer if the separation of the tunnel positions is sufficiently large. In the crossover regime one finds a rich behavior of the transmission probability due to multiple inelastic scattering and the interplay of the coherent tunnel motion and the ballistic particle motion. In general, for parameters outside the slow-tunneling regime the particle may systematically evade the scatterer so that its scattering strength is greatly reduced, and the transmission probability remains finite even for $U \rightarrow \infty$.

The quantum obstacle could be realized in double quantum dots $[1,2]$ or nanoconstrictions $[3,4]$. (A large interaction strength $U$ can be achieved in one-dimensional electronic transport because the particles cannot circumvent the interaction region.) As we will discuss at the end of the paper, the quantum delocalization of scatterers in such mesoscopic system should be experimentally detectable from the temperature dependence of the conductance.

In order to set the stage for the one-dimensional model system discussed below, let us first consider the "parallel" quantum scatterer in three dimensions, which hops between two positions $\boldsymbol{R}_{1}=\boldsymbol{r}_{0} / 2$ (internal state $|1\rangle$ ), $\boldsymbol{R}_{2}=-\boldsymbol{r}_{0} / 2$ (internal state $|2\rangle$ ), with separation $\boldsymbol{r}_{0}$. The eigenstates of the scatterer are the symmetric and antisymmetric combinations $|s\rangle=$ $2^{-1 / 2}(|1\rangle+|2\rangle)$ and $|a\rangle=2^{-1 / 2}(|1\rangle-|2\rangle)$, respectively. The corresponding eigenenergies $\mp \Delta / 2$ differ by the tunnel splitting $\Delta>0$. (As usual we assume that the ground state is the symmetric state $|s\rangle$.) This gives rise to tunneling of the scatterer between the two positions at a frequency $\nu=\Delta / h$. The incident particle with coordinate $\boldsymbol{r}$ interacts with the scatterer at position $\boldsymbol{R}_{k}$ through a potential $V_{k}(\boldsymbol{r})=V\left(\boldsymbol{r}-\boldsymbol{R}_{k}\right)$ which depends only on its relative position to the scatterer. The Hamiltonian describing the total system composed of the particle and 
the scatterer can then be written as

$$
\begin{aligned}
H & =\frac{\hat{\boldsymbol{p}}^{2}}{2 m}+\hat{W}-\frac{\Delta}{2}[|1\rangle\langle 2|+| 2\rangle\langle 1|], \\
\hat{W} & =V_{1}(\boldsymbol{r})|1\rangle\left\langle 1\left|+V_{2}(\boldsymbol{r})\right| 2\right\rangle\langle 2| .
\end{aligned}
$$

Here $\hat{\boldsymbol{p}}$ is the momentum operator of the particle of mass $m$. The plane-wave eigenstates $|\boldsymbol{k}\rangle$ are denoted by their wave vector $\boldsymbol{k}=\boldsymbol{p} / \hbar$.

We assume that the scatterer is initially prepared in its ground state $|s\rangle$ (preparation in its excited state $|a\rangle$ is equivalent to the case $\Delta<0$; superpositions result in nonstationary behavior). In the limit of a weak interaction we can apply the Born approximation and obtain the probability of scattering from the initial state $\left|\boldsymbol{k}_{\mathrm{i}}\right\rangle$ into the final state $\left|\boldsymbol{k}_{\mathrm{f}}\right\rangle$ by summing the probabilities for each final state of the scatterer:

$$
P_{\boldsymbol{k}_{\mathrm{i}} \rightarrow \boldsymbol{k}_{\mathrm{f}}}=\frac{2 \pi}{\hbar}\left\{\left|\left\langle\boldsymbol{k}_{\mathrm{f}} ; s|\hat{W}| s ; \boldsymbol{k}_{\mathrm{i}}\right\rangle\right|^{2} \delta\left(\varepsilon_{\mathrm{i}}-\varepsilon_{\mathrm{f}}\right)+\left|\left\langle\boldsymbol{k}_{\mathrm{f}} ; a|\hat{W}| s ; \boldsymbol{k}_{\mathrm{i}}\right\rangle\right|^{2} \delta\left(\varepsilon_{\mathrm{i}}-\varepsilon_{\mathrm{f}}-\Delta\right)\right\} .
$$

For a short-ranged potential of the form $V(\boldsymbol{r})=U \delta(\boldsymbol{r})$, the probability reads

$$
P_{\boldsymbol{k}_{\mathrm{i}} \rightarrow \boldsymbol{k}_{\mathrm{f}}}=\frac{2 \pi}{\hbar} U^{2}\left[\cos ^{2}\left(\Delta \boldsymbol{k} \cdot \boldsymbol{r}_{\mathbf{0}} / 2\right) \delta\left(\varepsilon_{\mathrm{i}}-\varepsilon_{\mathrm{f}}\right)+\sin ^{2}\left(\Delta \boldsymbol{k} \cdot \boldsymbol{r}_{\mathbf{0}} / 2\right) \delta\left(\varepsilon_{\mathrm{i}}-\varepsilon_{\mathrm{f}}-\Delta\right)\right],
$$

where $\Delta \boldsymbol{k}=\boldsymbol{k}_{\mathrm{i}}-\boldsymbol{k}_{\mathrm{f}}$. Interference with full contrast is observed when the energy $\varepsilon_{\mathrm{i}}$ of the incoming particle is smaller than $\Delta$ (fast tunneling), because then the argument of the second delta-function is always negative (inelastic processes are forbidden). In this situation the quantum scatterer acts as two classical scatterers of fixed positions $\boldsymbol{R}_{1}$ and $\boldsymbol{R}_{2}$, since $P_{\boldsymbol{k}_{\mathrm{i}} \rightarrow \boldsymbol{k}_{\mathrm{f}}} \sim$ $\cos ^{2}\left(\Delta \boldsymbol{k} \cdot \boldsymbol{r}_{0} / 2\right)$. On the other hand, interference is lost if the kinetic energy of the incident particles $\varepsilon_{\mathrm{i}} \gg \Delta$ (slow tunneling) [5]. The interference pattern can be recovered if one only detects the elastic scattering channel (by means of energy-resolved detection at energy $\varepsilon$ ).

Now we turn to the "serial" quantum barrier, which hops in the propagation direction of the scattered particle. In the case of one-dimensional scattering (plane-parallel barriers, or confined propagation) and for the delta-function potential $V_{1}(x)=U \delta(x-L / 2), V_{2}(x)=$ $U \delta(x+L / 2)$, this scattering problem can be solved exactly. (The problem is defined by the Hamiltonian given in eq. (1) with these potentials and $\hat{\boldsymbol{p}}^{2}$ replaced by $\hat{p}_{x}^{2}=-\hbar^{2} \partial^{2} / \partial x^{2}$.) In order to simplify the notation, we use units $\hbar^{2} / 2 m \equiv 1$, such that the kinetic energy $\varepsilon=k^{2}$.

Before we present the solution, let us briefly recall the results for the conventional case of immobile barriers. For a single immobile barrier of strength $U$ the transmission and reflection amplitudes at wave number $k$ are given by

$$
t=\frac{1}{1+i U / 2 k} \quad \text { and } \quad r=\frac{i U / 2 k}{1+i U / 2 k},
$$

respectively, such that the reflection probability approaches unity when $U \gg|k|$. When two such immobile barriers are placed in series with separation $L$ they form a Fabry-Perot interferometer, with transmission amplitude

$$
t=\frac{k^{2}}{k^{2}+i k U+U^{2}[\exp [2 i k L]-1] / 4} .
$$

For a large finesse of the interferometer $(U / k \gtrsim 1)$ one finds the well-known transmission resonances close to integer values of $k L / \pi$. 
The quantum scatterer is delocalized, giving rise to a number of additional resonance and interference effects. In order to explore these effects, we solve the stationary scattering problem for electrons with momentum $k>0$ incident from the left, while the scatterer is prepared in the eigenstate $|s\rangle$, hence giving the total energy $E=\varepsilon-\Delta / 2$. Under conservation of this energy, the electrons can be reflected or transmitted either elastically or inelastically, where in the latter case the outgoing electrons have momentum $\pm q$, with $q=\sqrt{\varepsilon-\Delta}$, and the scatterer is excited into the state $|a\rangle$.

The scattering probabilities and phase shifts can be determined via wave matching of the wave functions

$$
\phi_{\alpha}=\left(a_{\alpha} e^{i k x}+b_{\alpha} e^{-i k x}\right)|s\rangle+\left(c_{\alpha} e^{i q x}+d_{\alpha} e^{-i q x}\right)|a\rangle
$$

at the boundaries of the three regions $\alpha=\mathrm{I}$ for $x<-L / 2, \alpha=\mathrm{II}$ for $-L / 2<x<L / 2$, and $\alpha=$ III for $x>L / 2$ (see fig. 1). The resulting linear system of equations is then solved for $b_{\mathrm{I}}, d_{\mathrm{I}}, a_{\mathrm{III}}$, and $c_{\mathrm{III}}$ as a linear function of $a_{\mathrm{I}}$ (which we set to unity), under the conditions $c_{\mathrm{I}}=b_{\mathrm{III}}=d_{\mathrm{III}}=0$, because no electrons are coming in with momentum $q$, or from the right. (For the case $\varepsilon<\Delta$ we use the convention $\operatorname{Im} q>0$, so that the wave functions with coefficient $d_{\mathrm{I}}$ and $c_{\mathrm{III}}$ decay exponentially with the distance to the scatterer.) The coefficients (which are lengthy algebraic expressions and hence not written down here) determine the elastic and inelastic transmission and reflection probabilities by $T_{\mathrm{el}}=\left|a_{\mathrm{III}}\right|^{2}, T_{\text {inel }}=(\operatorname{Re} q / k)\left|c_{\mathrm{III}}\right|^{2}$, $R_{\mathrm{el}}=\left|b_{\mathrm{I}}\right|^{2}$, and $R_{\text {inel }}=(\operatorname{Re} q / k)\left|d_{\mathrm{I}}\right|^{2}$. (The inelastic scattering probability $T_{\text {inel }}+R_{\text {inel }}$

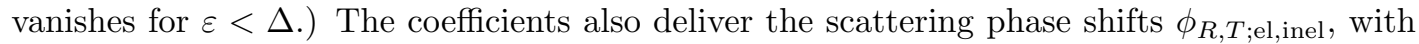
$\phi_{T ; \mathrm{el}}=\arg a_{\mathrm{III}}$, etc., and the delay times [6] $\tau=\hbar \mathrm{d} \phi / \mathrm{d} \varepsilon$. Having solved the problem in principle, let us now discuss the different regimes introduced in the introduction.

Slow tunneling. - In the limit $\Delta \rightarrow 0$ we find

$$
R=R_{\mathrm{el}}+R_{\text {inel }}=\frac{U^{2}}{4 k^{2}+U^{2}}, \quad R_{\mathrm{el}}=\cos ^{2}(k L) R, \quad T_{\mathrm{el}}=\frac{4 k^{2}}{4 k^{2}+U^{2}}, \quad T_{\text {inel }}=0 .
$$

The $L$-dependence of $R_{\mathrm{el}}$ corresponds to the result in Born approximation (3) with $\boldsymbol{k}_{\mathrm{i}} \| \boldsymbol{r}_{0}$ while $\boldsymbol{k}_{\mathrm{f}}$ points into the opposite direction, if we identify $\left|\boldsymbol{r}_{0}\right|=L$ and $|\Delta \boldsymbol{k}|=2 k$. Moreover, the total transmission and reflection probabilities are the same as for the conventional problem of a single delta-function of strength $U$ (see eq. (4)), and for $U \rightarrow \infty$ the transmittance vanishes. In striking contrast, we now will see that the transmission probability remains finite if $\Delta \neq 0$.

Crossover regime. - For $\Delta<\varepsilon$ we find for $U \rightarrow \infty$ the coefficients

$$
\begin{aligned}
& a_{\mathrm{III}}=2 i k g^{-1} \exp [i q L] \operatorname{Im} f, \quad c_{\mathrm{III}}=\exp [i(k-q) L / 2] a_{\mathrm{III}}, \\
& b_{\mathrm{I}}=2 q g^{-1}[i \operatorname{Im} f-k(\cos k L+\cos q L)], \\
& d_{\mathrm{I}}=k g^{-1} \exp [-i(k+q) L / 2][k(\exp [2 i q L]-1)+q(\exp [2 i k L]-1)],
\end{aligned}
$$

where $f=q \exp [i k L]-k \exp [i q L], g=(k+q)^{2}-f^{2}$. The proportionality between the transmission coefficients $a_{\mathrm{III}}$ and $c_{\mathrm{III}}$ in eq. (8) entails for the elastic and inelastic transmission delay times the relation $\tau_{T \text {;inel }}=\tau_{T ; \mathrm{el}}-m L\left(q^{-1}-k^{-1}\right) / 2 \hbar$, where we momentarily reintroduced the units $\hbar, m$.

The corresponding probabilities of transmission and inelastic scattering, as well as the delay times $\tau_{T \text {;el }}, \tau_{R \text {;el }}$, and $\tau_{R \text {;inel }}$, are plotted in fig. 2 as a function of $k L / \pi$ for fixed $\Delta / \varepsilon=0.4$. We find a regular sequence of transmission zeros, accompanied by long delay times for the various

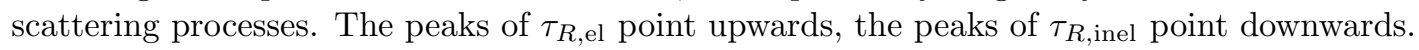




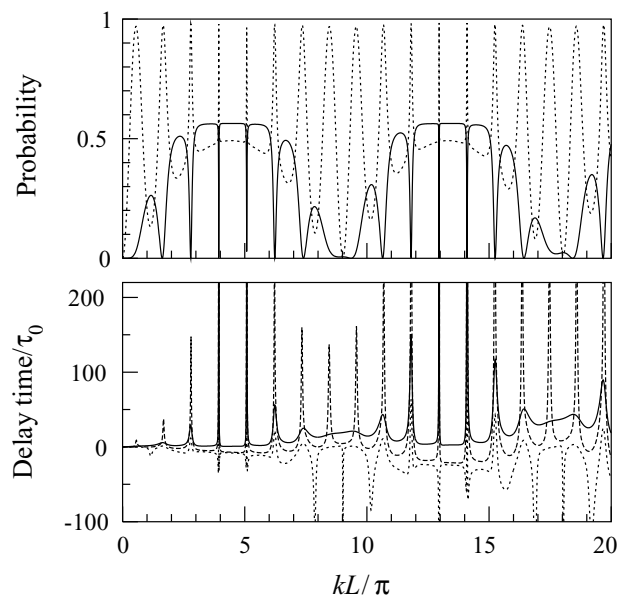

Fig. 2 - Upper panel: probabilities of transmission $T_{\mathrm{el}}+T_{\text {inel }}$ (solid curve) and inelastic scattering $T_{\text {inel }}+R_{\text {inel }}$ (dashed curve) for the one-dimensional model with $U=\infty$ as a function of $k L / \pi$, for $\Delta / \varepsilon=0.4$. Lower panel: delay times $\tau_{T ; \text { el }}$ (solid curve), $\tau_{R \text {;el }}$ (long dashes, peaks pointing upwards), and $\tau_{R ; \text { inel }}$ (short dashes, peaks pointing downwards), in units of $\tau_{0}=\hbar / \varepsilon$.

The transmission probability is modulated by a function $F(k L / \pi)$ (related to $f$ in eq. (8)) with period $p=2 /(1-\sqrt{1-\Delta / \varepsilon})$ and maxima at $k L / \pi=(n+1 / 2) p$. If we approach the slow-tunneling regime $(p \simeq 4 \varepsilon / \Delta)$, the maxima occur when the time of flight $L / v$ of the particle between the two positions of the obstacle is an odd multiple of the tunneling time $h / 2 \Delta$ between these positions (the minima occur at even multiples). Close to the minima the peaks in $\tau_{R, \mathrm{el}}$ and $\tau_{R \text {,inel }}$ alternate; close to the maxima they coincide.

At the border to the fast-tunneling regime $(\Delta=\varepsilon)$ the momentum $q$ vanishes, and the inelastic-scattering rate (which is proportional to Re $q$ ) drops to zero. The transmission probability becomes

$$
T=T_{\mathrm{el}}=\frac{(k L-\sin k L)^{2}}{(1+\cos k L)^{2}+(k L-\sin k L)^{2}} .
$$

In the limit $k L \gg 1$ the transmission probability $T=1$. This is a remarkable observation: The scatterer becomes totally transparent although $U \rightarrow \infty$.

Fast tunneling. - Another remarkable case of total transmission is found for $\Delta>\varepsilon$, where all electrons are scattered elastically. The transmission probability is now

$$
T=4 k^{2}(k \sinh |q| L-|q| \sin k L)^{2} e^{-2 L|q|}\left|(k+i|q|)^{2}-e^{i k L}\left(k e^{-L(|q|+i k)}-i|q|\right)^{2}\right|^{-2} .
$$

The delay times $\tau_{T}$ and $\tau_{R}$ are equal (this is a joint consequence of the unitarity of the scattering matrix and the reflection symmetry of the potential). At large length $L \gg 1 /|q|$ the transmission amplitude $t$ becomes exactly identical to the transmission amplitude of the Fabry-Perot interferometer, eq. (5), with $U$ replaced by $2|q|$. Hence, although we started out with an infinite scattering strength $U$, the finesse of the quantum version of the Fabry-Perot interferometer is finite.

The transmission probability and the delay time are plotted for $\Delta / \varepsilon=4$ as a function of $k L / \pi$ in fig. 3 (solid curves). The dashed curves show these quantities for two fixed classical barriers with scattering strength $U / k=2 \sqrt{3}$. The comparison demonstrates that the quan- 

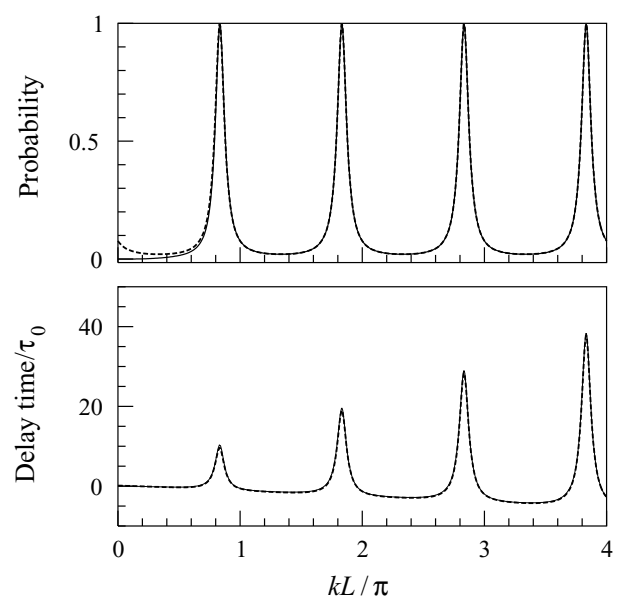

Fig. 3 - Transmission probability $T$ (upper panel) and delay time $\tau$ in units of $\tau_{0}=\hbar / \varepsilon$ (lower panel), as a function of $k L / \pi$. The solid curve is the result for the quantum obstacle with scattering strength $U=\infty$ and $\Delta / \varepsilon=4$, the dashed curve (nearly indistinguishable from the solid curve) the result for the conventional Fabry-Perot interferometer (two fixed classical barriers) from eq. (5) with $U / k=2 \sqrt{3}$.

tum obstacle behaves as two fixed classical scatterers when the tunnel splitting exceeds the kinetic energy.

It would be exciting to detect the quantum delocalization of scatterers in a mesoscopic system, e.g., by investigating the temperature dependence of the conductance. A possible manifestation of the delocalized scatterer in such systems is an interstitial defect, like a light atom, which hops between two energetically equivalent positions. When the thermal excitation energy is of the order of the energy barrier that the particle has to overcome, the defect jumps incoherently from one position to another. Since the potential in the system is changed after each jump, the conductance exhibits random temporal fluctuations (telegraphic noise) between two values $G_{1}$ and $G_{2}$ [7-9]. For a long measurement time one measures the time average $\langle G\rangle=\left(G_{1}+G_{2}\right) / 2$, which is small if the scattering strength is large. For lower temperatures, however, one enters the coherent-tunneling regime in which the defect acts as a quantum obstacle and its scattering strength can be greatly reduced, because it may be systematically avoided by the particles. Note that if electrons can be treated independently and scattering is elastic, our results for the transmission probability can be applied to obtain the conductance by the Landauer formula $G=\left(e^{2} / h\right) T$. Up to now, however, we have neglected many-body effects. The most straightforward modification is to account for the Pauli blocking of states below the Fermi energy $E_{\mathrm{F}}$ : Inelastic scattering is forbidden when the excitation energy $\varepsilon-E_{\mathrm{F}}<\Delta$. (The typical excitation energy is given by the potential drop $e V$ or by the thermal excitation energy, whatever the larger.) More intricate many-body effects arise from sequential scattering of several particles by the same quantum scatterer.

Can one also realize the serial quantum obstacle in one dimension (the Fabry-Perot interferometer)? An experimentally controllable set-up could consist of a single-channel wire placed adjacent to a double-quantum-dot device, which is tuned in resonance in the Coulombblockade regime. (For some experiments on double dots see refs. [1,2].) The quantum obstacle is the electron which occupies the two degenerate levels on the dots, and interacts with the electrons in the wire by Coulomb repulsion. In order to avoid complications from many-body 
effects to some extent, one might think of injecting "hot electrons" from one end of the wire, by shooting them over an additional potential barrier. In this way the excitation energies can be restricted to a small energy interval which is well separated from the Fermi energy.

To conclude, we have investigated scattering by a quantum obstacle which is delocalized in space. We found close analogies to the double slit and the Fabry-Perot interferometer for fast coherent tunneling of the obstacle, and that an infinitely high potential barrier can become transparent if it is delocalized. The dynamics of the delocalized scatterer is revealed in the efficiency with which the particle can evade the scatterer (the sensitive dependence of the effective scattering strength on kinetic energy and tunnel frequency).

Our results are relevant for recent experiments on mesoscopic structures that have probed the scattering by tunable $[1,2]$ or spontaneously formed $[3,4]$ two-level systems consisting of mobile entities. Moreover, experiments in molecular electronics revealed the interplay between electronic motion and kinetic degrees of freedom of the molecules [10]. The mobile entities and molecules are classical obstacles at high temperatures, but turn into quantum obstacles as the temperature is lowered. We have proposed a study of the temperature dependence of the conductance as a signature for having entered this new regime.

$$
* * *
$$

We thank S. Bahn, H. Bouchiat, A. Georges, Y. Imry, M. Kociak, and B. Reulet for discussions. This work was supported by the Dutch Science Foundation NWO/FOM and the European Commission.

\section{REFERENCES}

[1] Livermore C. et al., Science, 274 (1996) 1332.

[2] Fujisawa T. et al., Science, 282 (1998) 932.

[3] van den Brom H. E., Yanson A. I. and van Ruitenbeek J. M., Physica B, 252 (1998) 69.

[4] Keijsers R. J. P., Shklyarevskit O. I. and van Kempen H., Physica B, 253 (1998) 148.

[5] Cohen-Tannoudji C., Bardou F. and Aspect A., Laser Spectroscopy X, edited by Ducloy M., Giacobino E. and Camy G. (World Scientific, Singapore) 1992.

[6] Wigner E. P., Phys. Rev., 98 (1955) 145.

[7] Dutta P. and Horn P. M., Rev. Mod. Phys., 53 (1981) 497.

[8] Weissman M. B., Rev. Mod. Phys., 60 (1988) 537.

[9] Kogan Sh., Electronic Noise and Fluctuations in Solids (Cambridge University Press, Cambridge) 1996.

[10] PARK H. et al., Nature, 407 (2000) 57. 